more accurate predictions of developing or future crime patterns. This in turn can assist in planning for resource allocation-allowing police forces to move their officers from lower crime areas to places they may be more urgently needed. GIS can also be used as a community outreach tool for showing the public what their police forces are doing, and helping educate both neighborhood watch committees and individual citizens about what they can do to help protect themselves.

The book is geared toward the GIS beginner, but readers will still find having some GIS background very helpful. Most chapters feature two or three thematic scenarios followed by two or more Tutorials and a couple of Assignments each. The tutorials give step-by-step explanations for each of the scenarios covered, and also provide examples of the sort of results one should expect when performing, say, an attribute query. The assignments present similar tasks in a more "your turn now" manner, without the hand-holding. If the book were to be used as a classroom text, these assignments could easily become homework tasks to be handed in for grading. As with any other GIS book or tutorial, it is always best to complete all the steps in order.

The numerous maps and diagrams throughout the book are very helpful-especially for an individual without a GIS background-and can be used as a measuring stick that allows the reader to confirm that they are correctly completing and understanding the steps.

GIS Tutorial for Crime Analysis shows several ways that GIS can be applicable to police work. It introduces the basics of GIS to the neophyte, and is a great review for the GIS student going into the law enforcement field. I was taking my first course in GIS at the same time I was reviewing this book, and for the most part I had no problems or difficulties following it. I found each of the scenarios very interesting. I did find it a little disappointing, however, that in working through the Chapter 7 "Geocoding crime incident data" exercises, ArcMap would crash at various points-during both the Tutorial and Assignment segments. I retried the steps several times, and the same crashes would happen again and again. Some advanced GIS students in my school have since told me that they experience the same or similar problems with ArcMap in their coursework. It is not clear if this is a bug in the ArcMap program, or if the problem is in the tutorial, but either way, it disrupts the learning process.

Software problems aside, GIS Tutorial for Crime Analysis, Second Edition is a useful and usable resource for anyone interested in using GIS for crime analysis and police work. It explains the what and the why, and shows the how, of building a crime-analysis system, and it does so from the ground up for a reader with minimal pre-existing GIS skills.

\title{
A DIRECTORY OF CARTOGRAPHIC INVENTORS: CLEVER PEOPLE AWARDED A US PATENT FOR A MAP-RELATED DEVICE OR METHOD
}

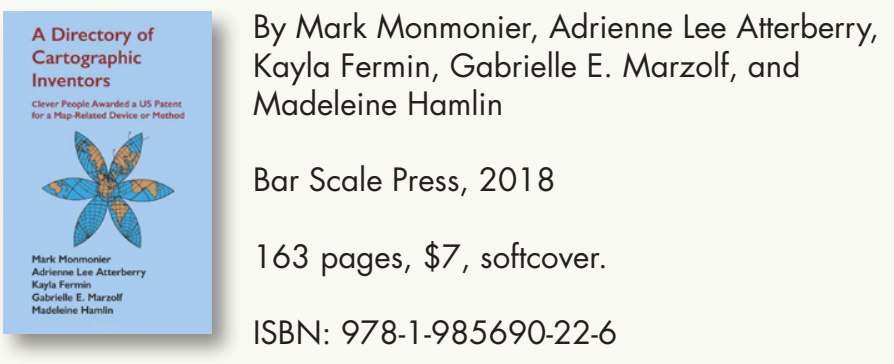

Review by: Trudy Suchan

A Directory of Cartographic Inventors provides biographical information about the cartographic patentees featured in Mark Monmonier's recently published Patents and Cartographic Inventions: A New Perspective for Map History (reviewed last year in Cartographic Perspectives 90). The
Directory is a resource that brings together and preserves much of the biographical research that underpinned, but did not make it into, Patents and Cartographic Inventions.

Where Patents and Cartographic Inventions is organized thematically around the inventions, $A$ Directory of Cartographic Inventors is a straight-up reference book with 31 biographies in alphabetical order. All but two of the patentees featured in the Directory's main section appear in Patents and Cartographic Inventions, as do all but three of the twenty-four additional inventors more briefly discussed at the back of the book.

The Directory covers a roughly two-hundred-year period between 1789 and 1995, from the birth of Silas Cornell 
(whose globe was patented in 1845) to the death of F. Webster McBryde (who patented his McBryde map projection in 1977).

The inventors profiled made their livings many ways but a few groupings emerge. Among the patentees that fit our contemporary definition of geographer are Henry de Beaumont, founder of the Geographic Society of Geneva and holder of a patent for a world projection. F. Webster McBryde was on the geography faculty at Ohio State University, served as a senior geographer in military intelligence at the War Department during World War II, and founded the American Society for Geographical Research. Alphons van der Grinten's patented map projection gained fame once the National Geographic Society took it up, albeit after his death. The number of educators and engineers who filed cartographic patents is also notable. Ellen Eliza Fitz and Elizabeth Oram-who each patented globes and are the only two women profiled-were in the education field. The Joneses (who patented an incar navigation system) and the Pollards (a map-quiz device) - two pairs of brothers - can be counted among the engineers. The book lists a number of patentees from the publishing and printing trades, and a great many that pursued multiple livelihoods. For example, John Plato (rural address finding) was a soldier, draftsman, lumber dealer, teacher, rancher, machinist, and a mapmaker for the US Government. Some, such as Edward Swett, were civic-minded, inventing a fire alarm system linked to a town or city street map. At least one inventor was rascally: Olin D. Gray (patentee of a globe containing a strip of pictures) ran an eponymous lithography firm and went afoul of the law by printing dodgy lottery tickets. Some sixty percent of those profiled held multiple patents, led by Jay Rhodes with over two hundred. Just shy of half of those listed in the Directory held additional patents unrelated to cartography; Max Bremsy, inventor of an in-car map system, also patented a cigar design.

The same template is applied across all of the biographies - birth date and place of origin, parents and siblings, schooling, means of livelihood and work locations, the patent(s), marriage and offspring, death date and placeand this structure makes clear the tantalizing gaps in any particular entry. Mark Monmonier's introduction catalogs the travails of searching for historical records and artifacts in today's online research domain. He and his team relied chiefly on census records and directories accessed through Ancestry Library Edition (ancestry.com/cs/us/ institution).

In the course of doing the research for a book or dissertation, one tends to become an expert on all manner of detail that does not, for one reason or another, fit the flow of the final work. A Directory of Cartographic Inventors: Clever People Awarded a US Patent for a Map-Related Device or Method is a model for preserving this kind of information in a form available to the cartographic community. And, it would be neatly circular if the biographical information in the Directory eventually filtered back into genealogy networks such as Ancestry.com.

\section{BEYOND THE MAP: UNRULY ENCLAVES, GHOSTLY PLACES, EMERGING LANDS, AND OUR SEARCH FOR NEW UTOPIAS}

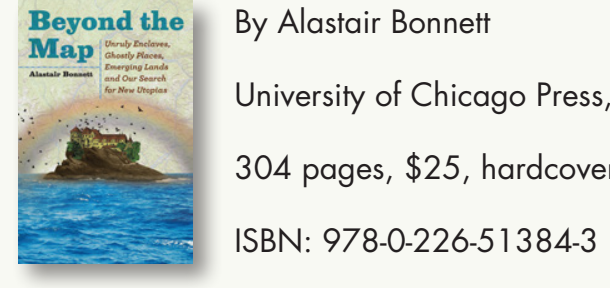

Review by: Nat Case, INCase, LLC

Beyond the Map is a collection of thirty-nine essays about places, regions, and geographic features, all in some way disputed, abandoned, transient, or otherwise resistant to "mappedness," though, disappointingly, maps themselves are not really discussed in the book. The chapters are grouped into five broad themes: "Unruly Islands," "Enclaves and Uncertain Nations," "Utopian Places," "Ghostly Places," and "Hidden Places," each of which is (broadly) self-explanatory. At the ostensible heart of the book is the vision of a centrifugal "new era of geographic giddiness" and fragmentation-a scenario wherein the author sees the old sensible order of the world unraveling in the new millennium. This central idea, however, does not provide a strong guiding principle for the book. Instead it's pervaded with a kind of exoticism, the sort that also runs through much of online site Atlas Obscura (atlasobscura. com). It is an updated, more socially conscious, and in this case somewhat weary spin on, Ripley's Believe It or Not. 